\title{
Efficient Implementation Algorithms for Homogenized Energy Models
}

\author{
Thomas R. Braun* and Ralph C. Smith ${ }^{\dagger}$ \\ Center for Research in Scientific Computation/Department of Mathematics \\ North Carolina State University, Raleigh, NC 27695
}

\begin{abstract}
The homogenized energy framework quantifying ferroelectric and ferromagnetic hysteresis is increasingly used for comprehensive material characterization and model-based control design. For operating regimes in which thermal relaxation mechanisms and stress-dependencies are negligible, existing algorithms are sufficiently efficient to permit device optimization and the potential for real-time control implementation. In this paper, we develop algorithms employing lookup tables which permit the high speed implementation of formulations which incorporate relaxation mechanisms and electromechanical coupling. Aspects of the algorithms are illustrated through comparison with experimental data.
\end{abstract}

\section{Homogenized Energy Framework}

Ferroelectric and ferromagnetic materials are being considered as transducers for an increasing number of applications due to their broadband capabilities, large electromechanical and magnetomechanical coupling factors, and their dual capability for actuating and sensing. At low drive levels, the direct and converse electromechani$\mathrm{cal} /$ magnetomechanical effects are approximately linear, and linear models and control designs can be employed. However, at the moderate to high drive levels where the unique transducer capabilities are manifested, the constitutive material properties are inherently nonlinear and hysteretic. Material characterization necessitates the development of models which accurately characterize constitutive nonlinearities and hysteresis whereas device optimization and real-time control implementation requires that the models be highly efficient to implement. While a number of frameworks have been developed for characterizing ferroelectric and ferromagnetic hysteresis, the competing requirements of accuracy and efficiency limit which models may be considered for both material characterization and real-time implementation.

At the microscopic scale, the physical mechanisms which produce hysteresis in ferroelectric and ferromagnetic materials differ substantially since ferroelectricity is due to the ionic structure of materials and ferromagnetism results from interactions between magnetic moments and electron spins. However, at the domain or macroscopic scale, shared physical and energy properties permit the development of unified frameworks for characterizing hysteresis of compounds (see [14] for details regarding shared properties of ferroic materials at the various scales).

In addition to the dielectric and magnetic hysteresis exhibited by the compounds, transducer models must characterize the thermal relaxation effects and stress-dependencies exhibited by ferroelectric and ferromagnetic materials. The former phenomenon is illustrated in Figure 1(a) with magnetic data collected from a steel rod [3]. Stress effects are illustrated in Figure 1(b) via PLZT data from [9].

As detailed in [14], several frameworks have been developed to characterize ferroelectric and ferromagnetic hysteresis for regimes in which relaxation mechanisms and stress-dependencies are negligible. These include

\footnotetext{
*Email: trbraun@ncsu.edu; Telephone: 919-854-2746
}

${ }^{\dagger}$ Email: rsmith@eos.ncsu.edu; Telephone: 919-515-7552 


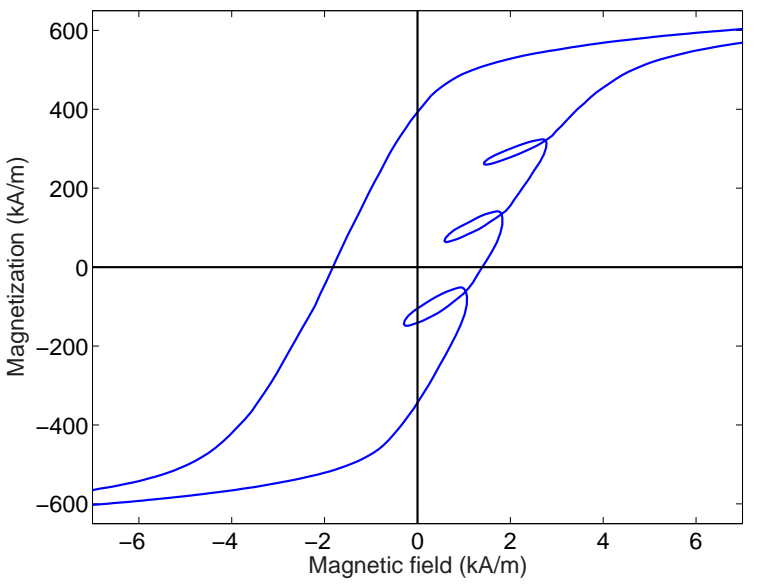

(a)

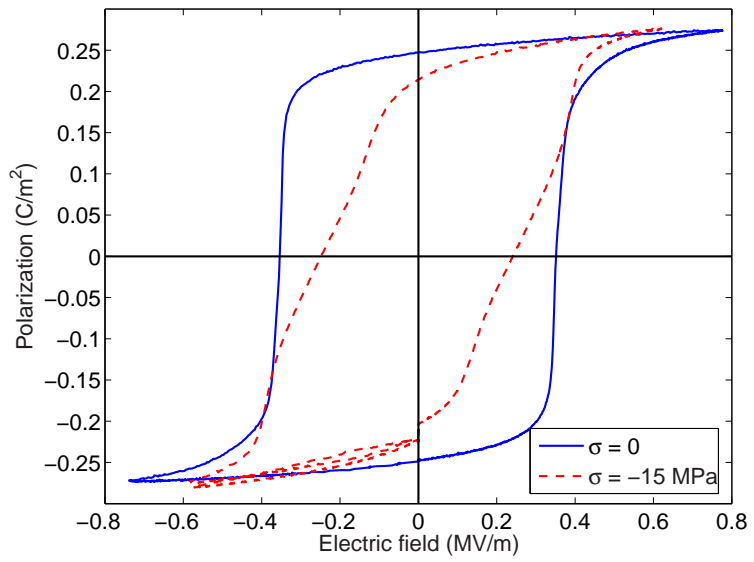

(b)

Figure 1: (a) Magnetization data from a steel rod illustrating the effect of magnetic after-effects on biased minor loops [3]. (b) PLZT data showing the effect of stress on polarization [9].

domain wall models [7, 10, 17], Preisach models [5, 12, 13, 21], and homogenized energy models [11, 15, 16, 18, 20]. Whereas certain facets of relaxation and stress-dependence have been incorporated in domain wall models and Preisach models - e.g., [4] and [6] - comprehensive theory incorporating these mechanisms for general compounds has not been developed within these two frameworks. Hence we focus on the homogenized energy framework which naturally incorporates thermal relaxation and direct electromechanical/magnetomechanical effects due to the energy basis of the framework.

As detailed in $[1,2,14,19,20]$, the general homogenized energy model for ferroelectric materials is

$$
[P(E, \sigma)](t)=\int_{0}^{\infty} \int_{-\infty}^{\infty}\left[\bar{P}\left(E+E_{I}, \sigma ; E_{c}\right)\right](t) \nu_{c}\left(E_{c}\right) \nu_{I}\left(E_{I}\right) d E_{I} d E_{c}
$$

where $E$ and $\sigma$ denote input electric fields and stresses, $P$ is the macroscopic polarization, $E_{c}$ and $E_{I}$ are local coercive and interaction fields, and $\nu_{c}, \nu_{I}$ are densities which incorporate the effects of material nonhomogeneities, polycrystallinity, and variable effective fields $E_{e}=E+E_{I}$. It is shown in subsequent sections that the kernel $\bar{P}$ follows from direct energy minimization when thermal relaxation effects are negligible or from Boltzmann principles if relaxation is significant.

The magnetic model is analogous. For magnetic field $H$, magnetization $M$, coercive field $H_{c}$ and interaction field $H_{I}$, the model is

$$
[M(H, \sigma)](t)=\int_{0}^{\infty} \int_{-\infty}^{\infty}\left[\bar{M}\left(H+H_{I}, \sigma ; H_{c}\right)\right](t) \nu_{c}\left(H_{c}\right) \nu_{I}\left(H_{I}\right) d H_{I} d H_{c} .
$$

In both cases, it is assumed that the densities $\nu_{c}$ and $\nu_{I}$ satisfy the criteria

$$
\begin{aligned}
& \nu_{c}(x) \text { defined for } x>0, \\
& \nu_{I}(-x)=\nu_{I}(x), \\
& \left|\nu_{c}(x)\right| \leq c_{1} e^{-a_{1} x}, \quad\left|\nu_{I}(x)\right| \leq c_{2} e^{-a_{2} x}
\end{aligned}
$$

for positive $c_{1}, a_{1}, c_{2}, a_{2}$. These assumptions enforce the physical properties that the local coercive fields are positive, low-field Rayleigh loops are symmetric, and local coercive and interaction fields decay as a function of distance.

For implementation purposes, either Gaussian or Newton-Cotes quadrature routines are used to approximate the integrals in (1) or (2) - see Chapter 8 of [14]. To illustrate, let $E_{c}[i], i=1, \ldots, N_{c}$, and $E_{I}[j], j=1, \ldots, N_{I}$, 
denote the abscissas (quadrature points) and $\widehat{w}_{c}[i], \widehat{w}_{I}[j]$ denote the respective quadrature weights. Approximation of the integrals in (1) then yields the discretized model

$$
\begin{aligned}
{[P(E, \sigma)](t) } & =\sum_{i=1}^{N_{c}} \sum_{j=1}^{N_{I}}\left[\bar{P}\left(E+E_{I}[j], \sigma ; E_{c}[i]\right)\right](t) \nu_{c}\left(E_{c}[i]\right) \nu_{I}\left(E_{I}[j]\right) \widehat{w}_{c}[i] \widehat{w}_{I}[j] \\
& =\sum_{i=1}^{N_{c}} \sum_{j=1}^{N_{I}}\left[\bar{P}\left(E+E_{I}[j], \sigma ; E_{c}[i]\right)\right](t) w_{c}[i] w_{I}[j]
\end{aligned}
$$

where $w_{c}[i]=\widehat{w}_{c}[i] \nu_{c}\left(E_{c}[i]\right)$ and $w_{I}[j]=\widehat{w}_{I}[j] \nu_{I}\left(E_{I}[j]\right)$. The discretization of the magnetization model is similar. We note that efficient implementation algorithms are required when large quadrature limits $N_{c}$ and $N_{I}$ are dictated by accuracy requirements.

The analogy between the polarization model (1) and magnetization model (2) illustrates the manner in which the framework characterizes hysteresis in the combined class of materials - see [14, 19]. To simplify subsequent discussion, we focus primarily on the polarization models (1) and (4) and note that analogous results follow for the ferromagnetic model. Aspects of both models are illustrated in examples and through comparison with experimental data in Section 5.

The technique for constructing the kernel $\bar{P}$ can be summarized as follows. We first construct Gibbs energy relations $G$ comprised of a Helmholtz energy $\psi$, which quantifies the internal energy associated with a continuum of dipole or moment configurations, and elastic, electrostatic, or magnetostatic work relations. For regimes in which thermal relaxation is negligible, $\bar{P}$ is determined by minimizing $G$ with respect to $P$. Physically, this can be interpreted as specifying the polarization that results when dipoles reorient in response to an applied field. To incorporate thermal relaxation mechanisms, the Gibbs and relative thermal energies are balanced using Boltzmann principles. As detailed in Section 2.6 of [14], this is equivalent to minimizing a combined measure of the internal and relative thermal energies.

In Section 2 we summarize the construction of appropriate energy functionals and formulation of the kernel $\bar{P}$. Whereas the model construction has appeared in the literature - e.g., see $[1,2,14,19]$ - we reformulate various components to facilitate the construction of highly efficient implementation algorithms through the use of lookup tables. These algorithms are presented in Section 3, and in Section 4 we illustrate that computation times can be reduced by up one to four of magnitude when implementing formulations which incorporate thermal relaxation and/or stress-dependencies. In Section 5 we illustrate the accuracy of the framework for characterizing the data in Figure 1.

\section{Energy Relations and Construction of the Kernel $\bar{P}$}

We summarize in Section 2.1 appropriate Helmholtz and Gibbs energy relations for stress-invariant regimes which involve $180^{\circ}$ switching and stress-dependent regimes with both $180^{\circ}$ and $90^{\circ}$ switching. In Section 2.2 we detail the manner through which the Gibb's energy is used to construct kernels $\bar{P}$ in the absence or presence of thermal activation which produces relaxation phenomenon. Issues relating to the implementation of the kernels are presented in Section 2.3, and various kernel constructions are compared from a computational perspective in Section 2.4.

\subsection{Helmholtz and Gibbs Energy Relations}

\subsubsection{Stress-Invariant Regimes: $180^{\circ}$ Switching}

As detailed in $[14,20]$, an appropriate Helmholtz energy relation for temperature-invariant, stress-invariant operating regimes with strictly $180^{\circ}$ switching is 


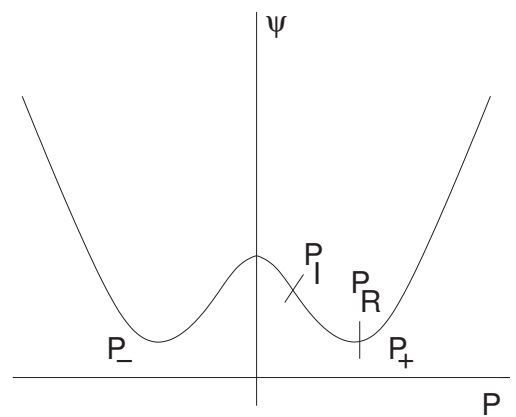

(a)

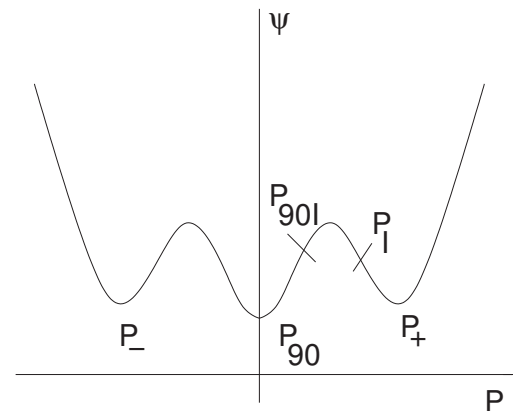

(b)

Figure 2: (a) Helmholtz energy for the stress-invariant case, and (b) Helmholtz energy for the stress-dependent case.

$$
\psi(P)=\left\{\begin{array}{ll}
\eta\left(P+P_{R}\right)^{2} / 2, & P \leq P_{I} \\
\frac{\eta}{2}\left(P_{I}-P_{R}\right)\left(\frac{P^{2}}{P_{I}}-P_{R}\right), & |P|<P_{I} \\
\eta\left(P-P_{R}\right)^{2} / 2, & P \geq P_{I}
\end{array},\right.
$$

where $P_{I}$ denotes the positive inflection point, $P_{R}$ is the local remanence value, and $\eta$ is the reciprocal slope $\frac{\partial E}{\partial P}$. It is shown in Section 2.2 that using this piecewise quadratic approximation for the energy results in a piecewise linear kernel where $\eta$ is the reciprocal slope of the positive and negative kernel branches. This energy relation is plotted in Figure 2(a).

The Helmholtz energy (5) quantifies the internal energy associated with positive and negative dipole orientations. The Gibbs energy relation

$$
G(E, P)=\psi(P)-E P
$$

balances this internal energy with the electrostatic energy or work performed by an applied external field. As detailed [14], where the Legendre transform properties of $G$ are discussed, care must be taken to interpret $E$ as the independent variable and $P$ as the dependent variable.

\subsubsection{Stress-Dependent Regimes: $180^{\circ}$ and $90^{\circ}$ Switching}

The Helmholtz relation (5) was derived under the assumption that dipoles are aligned either in the field direction or diametrically opposite to it - this characterizes the internal energy associated with field-induced $180^{\circ}$ switching. As illustrated in Figure 3, the application of stresses perpendicular to the poling direction additionally produces $90^{\circ}$ switching. It is illustrated in $[1,2]$ that whereas the strict incorporation of $90^{\circ}$ switching requires a $2-\mathrm{D}$ or 3-D energy landscape, reasonable approximations can be obtained for a number of applications with 1-D Helmholtz relations whose minima correspond with $180^{\circ}$ and $90^{\circ}$ dipole positions, as shown in Figure 2(b). We summarize this latter case.

Following the development in $[1,2]$, the Helmholtz energy quantifying the internal energy associated with the three dipole states is taken to be

$$
\psi_{p}(P)= \begin{cases}\eta\left(P+P_{R}\right)^{2} / 2, & P \leq-P_{I} \\ \eta_{1}\left(P+P_{m}\right)^{2} / 2+\beta, & -P_{I}<P<-P_{90 I} \\ \eta_{2} P^{2} / 2+\Delta, & |P|<P_{90 I} \\ \eta_{1}\left(P-P_{m}\right)^{2} / 2+\beta, & P_{90 I}<P<P_{I} \\ \eta\left(P-P_{R}\right)^{2} / 2, & P \leq-P_{I}\end{cases}
$$




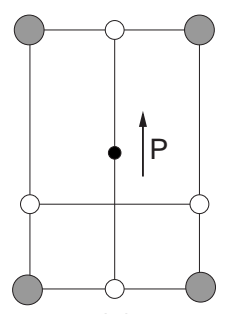

(a)

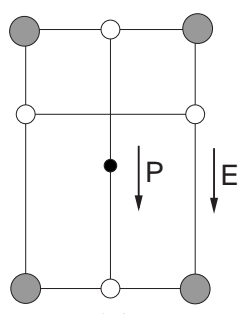

(b)

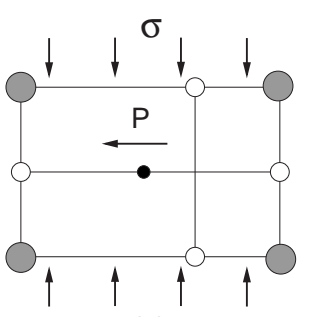

(c)

Figure 3: Possible polarization states of a single crystal including (a) the state with 0 applied field, (b) the $180^{\circ}$ switch which occurs with a sufficiently strong negative field, and (c) a $90^{\circ}$ switch induced by stress.

where

$$
\begin{array}{cc}
P_{m}=\frac{\eta\left(P_{I}-P_{R}\right) P_{90 I}-\eta_{2} P_{90 I} P_{I}}{\eta\left(P_{I}-P_{R}\right)-\eta 2 P_{90 I}}, & \eta_{1}=\eta \frac{P_{I}-P_{R}}{P_{I}-P_{m}} \\
\beta=\frac{\eta}{2}\left(P_{I}-P_{R}\right)^{2}-\frac{\eta_{1}}{2}\left(P_{I}-P_{m}\right)^{2}, & \Delta=\frac{\eta_{1}}{2}\left(P_{90 I}-P_{m}\right)^{2}+\beta-\frac{\eta_{2}}{2} P_{90 I}^{2} .
\end{array}
$$

The parameters $P_{90 I}$ and $\eta_{2}$ are analogous to $P_{I}$ and $\eta$, except they apply to dipoles that are normal to the applied field (i.e., in a $90^{\circ}$ orientation). Note that due to the one dimensional context, we consider all normal directions as the same state. This distinguishes this from a true 2-D approach but greatly simplifies the computation.

The energy relation (7) incorporates the internal energy associated with dipole switching but neglects electromechanical coupling and elastic contributions. Electromechanical coupling can be quantified by the relation

$$
\psi_{e s}(P, \varepsilon)=-a \varepsilon P-q \varepsilon P^{2}
$$

where $a$ is the piezoelectric coupling coefficient, $q$ is the electrostrictive coupling coefficient, and $\varepsilon$ is the strain. The elastic energy for uniaxial regimes is

$$
\psi_{e l}(\varepsilon)=\frac{1}{2} Y \varepsilon^{2}
$$

where $Y$ is the Young's modulus of the material. The total Helmholtz energy is thus

$$
\psi(P, \varepsilon)=\psi_{p}(P)+\psi_{e s}(P, \varepsilon)+\psi_{e l}(\varepsilon) .
$$

The associated Gibb's energy is

$$
G(E, P, \sigma, \epsilon)=\psi(P, \varepsilon)-E P-\sigma \varepsilon
$$

where $\sigma$ is the stress. As detailed in $[1,2]$, enforcement of the quilibrium condition $\frac{\partial G}{\partial \varepsilon}=0$ allows the strain to be written as

$$
\varepsilon=Y^{-1}\left(\sigma+a P+q P^{2}\right) .
$$

Substituting into (11) yields a Gibb's formulation posed solely in terms of the input field and stress.

\subsection{Relations for the kernel $\bar{P}$}

\subsubsection{Stress-Invariant Regimes: $180^{\circ}$ Switching}

For regimes in which thermally activated relaxation is negligable, direct minimization of (6) yields the kernel

$$
\bar{P}=\frac{E}{\eta}+P_{R} \delta
$$


where $\delta=1$ for positively oriented dipoles and $\delta=-1$ for negatively oriented dipoles. From (13), it follows that the local coercive field $E_{c}$, local remanence polarization $P_{R}$, and inflection point $P_{I}$ are related by the expression

$$
P_{I}=P_{R}-\frac{E_{c}}{\eta} .
$$

Thermal activation is manifested by dipoles having sufficient thermal energy to switch states before a minima of the Gibbs energy is eliminated. To quantify this, the Boltzmann relation

$$
\mu(G)=C \exp (-G(E, P) V / k T)
$$

balances the Gibbs and relative thermal energies, where $V$ is a reference volume, $T$ is the temperature in degrees Kelvin, and $k$ is Boltzmann's constant. The constant $C$ is a chosen to ensure integration to unity. It is shown in $[14,20]$ that the resulting kernel is

$$
\bar{P}=x_{+}\left\langle P_{+}\right\rangle+\left(1-x_{+}\right)\left\langle P_{-}\right\rangle
$$

where $x_{+}$denotes the fraction of positively oriented dipoles and

$$
\left\langle P_{+}\right\rangle=\frac{\int_{P_{I}}^{\infty} P \exp \left(\frac{-G(E, P) V}{k T}\right) d P}{\int_{P_{I}}^{\infty} \exp \left(\frac{-G(E, P) V}{k T}\right) d P} \quad,\left\langle P_{-}\right\rangle=\frac{\int_{-\infty}^{-P_{I}} P \exp \left(\frac{-G(E, P) V}{k T}\right) d P}{\int_{-\infty}^{-P_{I}} \exp \left(\frac{-G(E, P) V}{k T}\right) d P}
$$

are the average polarizations associated with positive and negative dipole orientations. The evolution of dipole fractions is governed by the differential equation

$$
\dot{x}_{+}=-p_{+-} x_{+}+p_{-+}\left(1-x_{+}\right),
$$

where the likelihoods $p_{+-}$and $p_{-+}$of a dipole switching from positive to negative, or vice-versa, are

$$
p_{+-}=\frac{\int_{P_{I}}^{P_{I}+\epsilon} \exp \left(\frac{-G(E, P) V}{k T}\right) d P}{\tau(T) \int_{P_{I}}^{\infty} \exp \left(\frac{-G(E, P) V}{k T}\right) d P}, \quad p_{-+}=\frac{\int_{-P_{I}-\epsilon}^{-P_{I}} \exp \left(\frac{-G(E, P) V}{k T}\right) d P}{\tau(T) \int_{-\infty}^{-P_{I}} \exp \left(\frac{-G(E, P) V}{k T}\right) d P} .
$$

Here $\tau$ is the material and temperature-dependent relaxation time and $\epsilon$ is a small, positive constant (its purpose is detailed in Section 2.3.1).

For implementation purposes, a backward Euler discretization can provide sufficient accuracy when solving (18) if stepsizes are reasonably small. The integrals in (17) and (19) can be solved via quadrature routines. However, this is not necessary in this case, since it is shown in Section 2.3.2 that they can be formulated as a combination of exponential and error functions. These functions are provided by most mathematical libraries and generally provide better performance than direct evaluation in both computation time and accuracy.

\subsubsection{Stress-Dependent Regimes: $180^{\circ}$ and $90^{\circ}$ Switching}

The kernel development for the stress-dependent case is analogous but employs the Gibbs relation (11). Due to the definition (7) in the Helmholtz energy, 4 switching points occur: from either positive or negative to $90^{\circ}$ and from $90^{\circ}$ to either $180^{\circ}$ orientation. We let $E_{c}$ denote the field at which the polarization switches to a positive orientation and specify the transition to a ninety degree orientation through the independent parameter $P_{I}$. The input electric field levels at which the dipole switches to $90^{\circ}$ orientations occur at

$$
\begin{aligned}
& E_{+90}=\frac{-1}{Y}\left(2 q^{2} P_{I}^{3}+3 a q P_{I}^{2}+\left(a^{2}-\eta Y+2 q \sigma\right) P_{I}+a \sigma+\eta Y P_{R}\right), \\
& E_{-90}=\frac{1}{Y}\left(2 q^{2} P_{I}^{3}-3 a q P_{I}^{2}+\left(a^{2}-\eta Y+2 q \sigma\right) P_{I}-a \sigma-\eta Y P_{R}\right) .
\end{aligned}
$$

Note that this implies $180^{\circ}$ switches will always be symmetric whereas if linear electromechanical coupling is significant, the $90^{\circ}$ switches may not be symmetric. This was chosen to match behavior observed in stressed ferroelectric materials. 
For regimes in which thermal activation is negligible, the roots of $\frac{\partial G}{\partial P}$ may be obtained numerically for each of the cases $P \geq P_{I}, P \leq-P_{I}$ and $|P| \leq P_{90 I}$. While it is possible that more than one real root may exist, only one is typically within a valid range for $P$.

To incorporate thermal activation, we again balance (11) with relative thermal energy via the Boltzmann relation (15). This yields three average polarization relations

$$
\begin{gathered}
\left\langle P_{+}\right\rangle=\frac{\int_{P_{I}}^{\infty} P \exp \left(\frac{-G(E, P) V}{k T}\right) d P}{\int_{P_{I}}^{\infty} \exp \left(\frac{-G(E, P) V}{k T}\right) d P}, \quad\left\langle P_{-}\right\rangle=\frac{\int_{-\infty}^{-P_{I}} P \exp \left(\frac{-G(E, P) V}{k T}\right) d P}{\int_{-\infty}^{-P_{I}} \exp \left(\frac{-G(E, P) V}{k T}\right) d P} \\
\left\langle P_{90}\right\rangle=\frac{\int_{-P_{90 I}}^{P_{90 I}} P \exp \left(\frac{-G(E, P) V}{k T}\right) d P}{\int_{-P_{90 I}}^{P_{90 I}} \exp \left(\frac{-G(E, P) V}{k T}\right) d P}
\end{gathered}
$$

corresponding to the three dipole states. Since dipoles have three orientations, we must keep track of the fraction oriented positively $x_{+}$and negatively $x_{-}$. The third fraction is given by $x_{90}=1-x_{+}-x_{-}$. This resulting kernel is

$$
\bar{P}=x_{+}\left\langle P_{+}\right\rangle+x_{-}\left\langle P_{-}\right\rangle+\left(1-x_{+}-x_{-}\right)\left\langle P_{90}\right\rangle .
$$

The evolution of dipole fractions is simplified by assuming that a dipole may only switch to an adjacent state; i.e., a negative dipole must switch to $90^{\circ}$, not directly to positive. This yields the likelihood relations

$$
\begin{array}{cc}
p_{+90}=\frac{\int_{P_{I}}^{P_{I}+\epsilon} \exp \left(\frac{-G(E, P) V}{k T}\right) d P}{\tau(T) \int_{P_{I}}^{\infty} \exp \left(\frac{-G(E, P) V}{k T}\right) d P}, & p_{-90}=\frac{\int_{-P_{I}-\epsilon}^{-P_{I}} \exp \left(\frac{-G(E, P) V}{k T}\right) d P}{\tau(T) \int_{-\infty}^{-P_{I}} \exp \left(\frac{-G(E, P) V}{k T}\right) d P}, \\
p_{90+}=\frac{\int_{P_{90 I}-\epsilon}^{P_{90 I}} \exp \left(\frac{-G(E, P) V}{k T}\right) d P}{\tau(T) \int_{-P_{90 I}}^{P_{90 I}} \exp \left(\frac{-G(E, P) V}{k T}\right) d P}, & p_{90-}=\frac{\int_{-P_{90 I}}^{-P_{90 I}+\epsilon} \exp \left(\frac{-G(E, P) V}{k T}\right) d P}{\tau(T) \int_{-P_{90 I}}^{P_{90 I}} \exp \left(\frac{-G(E, P) V}{k T}\right) d P}
\end{array}
$$

and the differential equation

$$
\left[\begin{array}{c}
\dot{x_{-}} \\
x_{+}
\end{array}\right]=\left[\begin{array}{cc}
-p_{-90}-p_{90-} & -p_{90-} \\
-p_{90+} & -p_{+90}-p_{90+}
\end{array}\right]\left[\begin{array}{l}
x_{-} \\
x_{+}
\end{array}\right]+\left[\begin{array}{l}
p_{90-} \\
p_{90+}
\end{array}\right]
$$

governing the evolution of dipole fractions. Again, a backward Euler method typically provides sufficient accuracy while minimizing computation. We note that unlike the stress-free case, the integrals in (21) and (23) cannot be reduced to error functions and exponentials and hence must be approximated with a quadrature method.

\subsection{Theoretical and Practical Implementation Details}

We summarize here issues pertaining to the construction of components arising in the kernel definitions and implementations.

\subsubsection{Construction of Likelihood Relations}

The relations (19) and (23) quantify the likelihood that dipoles achieve the relative thermal energy required to reorient in advance of fields required to eliminate minima of the Gibbs energy. As detailed in Section 2.6 of [14], this is initially formulated as a sum of the discrete set of dipole states with the continuum assumption and subsequent integration performed to facilitate evaluation of the likelihoods. For the discrete set of states, the likelihoods are computed by point evaluation of $G$ at $P_{I}$. Under the assumption of a continuum, however, point evaluation yields probabilities and hence likelihoods of 0 . Thus, care must be taken when formulating appropriate likelihood relations.

One approach is to employ the formulations (19) and (23) where $\epsilon$ is chosen to be a small positive constant. The resulting likelihood relations are well-posed but have the disadvantage of requiring an additional, nonphysical parameter $\epsilon$. 
Alternatively, one can approximate the integrals in (19) and (23) with Riemann sums and incorporate $\epsilon$ into the relaxation time $\tau$ to obtain the expressions

$$
p_{+-}=\frac{\exp \left(\frac{-G\left(E, P_{I}\right) V}{k T}\right)}{\tau(T) \int_{P_{I}}^{\infty} \exp \left(\frac{-G(E, P) V}{k T}\right) d P}, \quad p_{-+}=\frac{\exp \left(\frac{-G\left(E,-P_{I}\right) V}{k T}\right)}{\tau(T) \int_{-\infty}^{-P_{I}} \exp \left(\frac{-G(E, P) V}{k T}\right) d P} .
$$

This avoid the uncertainty in the choice of $\epsilon$ and is advantageous in 2-D and 3-D models where the integrals become surface or volume integrals. For stress-invariant one-dimensional models, both likelihood relations may be expressed in terms of the complementary error function. While either approach is reasonable and yields similar results, we employ the former approach in subsequent algorithms.

Either approach for determining likelihood may still yield the nonphysical situation that a dipole has not switched even after its input $E_{e}$ has passed the coercive point. The likelihood calculation is intended to allow dipoles to switch ahead of this point. However, for some parameter values it may dictate that dipoles attempt to switch infrequently, possibly not until after the field has passed the coercive point. To remove this possibility, we employ (19) only when $E_{e}<E_{c}$ (for $p_{-+}$) or $E_{e}>-E_{c}$ (for $p_{+-}$). For other values, the likelihood is defined to be $\frac{1}{\tau(T)}$. Analogous definitions are employed for (23). This also has a computational advantage, as (19) or (23) may overflow the floating point number system for values of $E_{e}$ well beyond the coercive point. This definition removes these floating point overflows from consideration.

\subsubsection{Conversion of Integrals to Exponentials and Error Functions}

In the stress-invariant thermal activation case, the integrals in (17) and (19) can be expressed in terms of exponential and complementary error functions to simplify their computation. For brevity, this will only be derived for the positive likelihood and average polarization. The computation of negative likelihood and average polarization relations is analogous. Consider the integral

$$
\int_{x}^{y} \exp \left(-\frac{G V}{k T}\right)=\int_{x}^{y} \exp \left(-\frac{V}{k T}\left(\frac{\eta}{2}\left(P-P_{R}\right)^{2}\right)-E P\right) .
$$

This can be rewritten as

$$
\begin{aligned}
\alpha \int_{x}^{y} & \exp \left(-\frac{\eta V}{2 k T}\left(P-\left(P_{R}+\frac{E}{\eta}\right)\right)^{2}\right) d P \\
& =\alpha \int_{\left(x-P_{R}-E_{e} / \eta\right) \sqrt{\eta V / 2 k T}}^{\left(y-P_{R}-E_{e} / \eta\right) \sqrt{\eta V / 2 k T}} \exp \left(-t^{2}\right) d t \\
& =\alpha \sqrt{\frac{2 k T}{\eta V}}\left[\operatorname{erfc}\left(\left(x-P_{R}-\frac{E}{\eta}\right) \sqrt{\frac{\eta V}{2 k T}}\right)-\operatorname{erfc}\left(\left(y-P_{R}-\frac{E}{\eta}\right) \sqrt{\frac{\eta V}{2 k T}}\right)\right]
\end{aligned}
$$

where $\alpha=\exp \left(P_{R}^{2}-\left(P_{R}+\frac{E}{\eta}\right)^{2}\right)$. Applying this to the likelihood definition and substituting for $P_{I}$ yields

$$
p_{+-}=\frac{1}{\tau}\left[1-\frac{\operatorname{erfc}\left(\left(-\frac{E_{c}}{\eta}-\frac{E}{\eta}+\epsilon\right) \sqrt{\frac{\eta V}{2 k T}}\right)}{\operatorname{erfc}\left(\left(-\frac{E_{c}}{\eta}-\frac{E}{\eta}\right) \sqrt{\frac{\eta V}{2 k T}}\right)}\right] .
$$

To specify the average polarization, we note that

$$
\int_{P_{I}}^{\infty} P \exp \left(-\frac{G V}{k T}\right) d P=\alpha \int_{P_{I}}^{\infty} P \gamma d P=-\frac{\alpha k T}{\eta V} \int_{P_{I}}^{\infty} \frac{-\eta V}{k T}\left(P-P_{R}-\frac{E}{\eta}\right) \gamma d P+\alpha\left(P_{R}+\frac{E}{\eta}\right) \int_{P_{I}}^{\infty} \gamma d P
$$


where $\gamma=\exp \left(-\frac{\eta V}{2 k T}\left(P-P_{R}-\frac{E}{\eta}\right)^{2}\right)$. The first integrand is now the derivative of $\gamma$ which yields

$$
\left\langle P_{+}\right\rangle=\sqrt{2}\left(\frac{\eta V}{k T}\right)^{\frac{3}{2}}\left[\frac{\exp \left(-\left(-\frac{E_{c}}{\eta}-\frac{E}{\eta}\right) \frac{\eta V}{2 k T}\right)}{\operatorname{erfc}\left(\left(-\frac{E_{c}}{\eta}-\frac{E}{\eta}\right) \sqrt{\frac{\eta V}{2 k T}}\right)}\right]+\frac{E}{\eta}+P_{R} .
$$

We note that this re-formulation is not possible in the stress-dependent case due to the additional terms in (11).

\subsubsection{Quadrature Methods and Stress-Dependence}

In addition to requiring quadrature methods to evaluate (21) and (23), stress-dependence also necessitates care when choosing the quadrature method used to approximate the coercive field integral in (4). Note that $\nu_{c}(x)$ is defined only for $x>0$. Some quadrature methods for this distribution - such as a trapezoid or Simpson's rule - will include the zero endpoint. As long as $\nu_{c}(0)=0$, this poses no issue. However, if $\nu_{c}(0) \neq 0$, the stress-invariant formulations will still yield reasonable results. In the stress-dependent formulation, $\nu_{c}(0) \neq 0$ gives dipoles whose coercive point from $90^{\circ}$ to positive is the same as from $90^{\circ}$ to negative, i.e., dipoles try to switch both directions at once. This yields nonphysical and numerically ambiguous results. For this reason, implementation of the stress-dependent formulation must avoid $\nu_{c}$ quadrature points at 0 either by enforcing $\nu_{c}(0)=0$ or by employing a quadrature method that does not use the left endpoint. Gaussian quadrature is a natural choice for this reason as well as for accuracy.

\subsubsection{Avoidance of Overflow}

A final note regards the calculation of (21) and (23). Whereas the equations themselves are well-defined, the numerators and denominators may grow quite large or small, leading to either a numerical overflow or underflow/division by zero. This occurs more frequently for large $V / k T$. As discussed in [14], the activation behavior approaches the negligible relaxation model as $V / k T \longrightarrow \infty$. Thus, overflow is dealt with by substituting the limit - the same energy minimization performed in the negligible activation case - whenever it occurs.

\subsection{Numerical Cost of Kernels}

The run-times for algorithms directly implementing the kernels $\bar{P}$ specified in (13), (16) and (22) are summarized in Table 1. We note that inclusion of stress-dependence results in an algorithm that runs approximately 25 times slower whereas the inclusion of thermal relaxation requires at least 90 times more computational effort. In the worst case, inclusion of both relaxation and stress-dependence is over 28,000 times slower than assuming both are negligible.

It should be emphasized that the run-time depends greatly on several implementation criteria. For example, in the stress-dependent negligible relaxation case, the roots of a cubic polynomial must be computed. These may be computed through equations for cubic polynomials or through eigenvalue calculation (the MATLAB roots command employs the latter approach). The choice of method will impact the run-time but does not change the qualitative difference in computation time between formulations.

\begin{tabular}{lc}
\hline Implementation Algorithm & Run-time $(\mathrm{s})$ \\
\hline stress-invariant, negligible relaxation & $2.758 \times 10^{1}$ \\
stress-invariant, relaxation & $2.448 \times 10^{3}$ \\
stress-dependent, negligible relaxation & $6.751 \times 10^{2}$ \\
stress-dependent, relaxation & $7.880 \times 10^{5}$ \\
\hline
\end{tabular}

Table 1: Run-times employing a direct implementation the kernels $\bar{P}$ specified in (13), (16) and (22). Run-times are for 120,000 temporal iterations and 80 quadrature points for each of the integrals in (4). 


\section{Look-up Tables for Faster Computation}

It has been demonstrated that inclusion of stress-dependencies or thermal relaxation mechanisms increases the computation effort (defined as floating point operations) by over an order of magnitude in all cases. For many control systems, this will preclude the possibility of real-time implementation. To improve efficiency, the model can be approximated to within an arbitrary degree of accuracy by employing look-up tables. This effectively trades memory for computation time. The necessary lookup tables will be derived for thermal relaxation, stressdependence, and combined relaxation and stress-dependent formulations.

\subsection{Algorithm for Negligible Relaxation, Stress-Invariant Regimes}

Regimes with negligible relaxation and stresses do not require lookup tables. However, the computation time may be halved through algorithm optimizations. Note that the discretized model may be written as

$$
\begin{aligned}
P & =\sum_{i=1}^{N_{c}} \sum_{j=1}^{N_{I}} w_{c}[i] w_{I}[j]\left(\frac{E}{\eta}+\frac{E_{I}[j]}{\eta}+P_{R} \delta[i, j]\right) \\
& =\frac{E}{\eta} \sum_{i=1}^{N_{c}} \sum_{j=1}^{N_{I}} w_{c}[i] w_{I}[j]+\sum_{i=1}^{N_{c}} \sum_{j=1}^{N_{I}} w_{c}[i] w_{I}[j] \frac{E_{I}[j]}{\eta}+\sum_{i=1}^{N_{c}} \sum_{j=1}^{N_{I}} w_{c}[i] w_{I}[j] P_{R} \delta[i, j] .
\end{aligned}
$$

In this form, the first term is a constant times the input field, whereas the second is constant. Only the third term will depend on each quadrature point. Premultiplying either $w_{c}$ or $w_{I}$ by $P_{R}$ further reduces the number of computations per time step. These changes are reflected in Algorithm 1. Similar optimizations were performed for each of the following formulations.

\subsection{Algorithm for Thermal Relaxation, Stress-Invariant Regimes}

As shown in Section 2.4, the majority of the computational effort for the stress-invariant relaxation model is spent in the computation of the average polarization and likelihood relations (17) and (19). If it were possible to calculate and store these values in advance, the per iteration time would decrease significantly. The input field $E$ is bounded by physical constraints whereas the quadrature points must also be bounded due to the decay of $\nu_{c}$ and $\nu_{I}$ as posited in (3). Thus, the values associated with each exp and erfc evaluation are bounded and this bound can be determined a priori. This defines the lookup table approach; before running the algorithm, the range between each input is discretized, and the values of (17) and (19) are computed and stored for every grid element. With even a moderate number of time steps, this reduces the overall computational effort; however, the
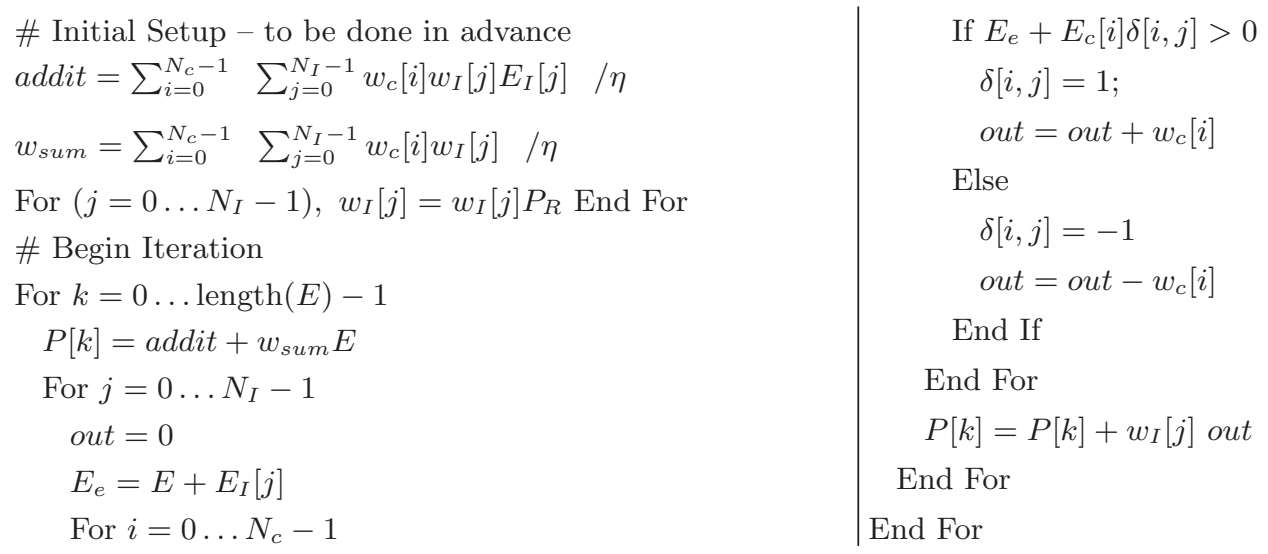

Algorithm 1: Implementation algorithm for regimes with negligible relaxation and no applied stress. 
main purpose is to replace computationally intense real-time components of the algorithm by offline computations and efficient memory usage.

It may appear that the resulting grid is three dimensional with one dimension for each of the input field $E$, interaction field $E_{I}$ and coercive field $E_{c}$. However, $E$ and $E_{I}$ are related by $E_{e}=E+E_{I}$ and as such will always share a single dimension in the grid. Further, these variables always occur as the combination

$$
E_{t m p-}=E+E_{I}-E_{c}
$$

in the relations for $\left\langle P_{-}\right\rangle$and $p_{-+}$whereas for $\left\langle P_{+}\right\rangle$and $p_{+-}$the variables occur as

$$
E_{t m p+}=E+E_{I}+E_{c} .
$$

We therefore compute the bounds of $E_{t m p-}$ and $E_{t m p+}$, discretize these variables, and calculate four 1-D tables of values, one for each of $\left\langle P_{-}\right\rangle, p_{-+},\left\langle P_{+}\right\rangle$and $p_{+_{-}}$. Further memory can be saved by noting that the likelihoods need not be calculated for values of $E_{t m p-}>0$ or $E_{t m p+}<0$ as they are defined to be constant in these cases (see Section 2.3). Pseudocode for this formulation is given in Algorithm 2.

It should be noted that the likelihoods and average polarizations need not use the same discretization of $E_{t m p-}$ and $E_{t m p+}$; in fact, the best accuracy for a given memory usage can be obtained by storing more points for the likelihoods than for the average polarizations. This is due to the likelihoods being exponential functions whereas the average polarizations are nearly linear.

Memory usage and algorithmic accuracy, defined as the difference between the lookup table approximation and a direct model implementation, are linearly related. However, the number of computations performed by the real-time portion algorithm is independent of the lookup table size; the difference is completely contained in the offline setup.

\subsection{Algorithm for Stress-Dependent, Negligible Relaxation Regimes}

When stress-dependence is considered in the absence of relaxation, there are no likelihoods to compute. However, minimizing (11) requires computing zeros of a cubic polynomial. Given the piecewise definition of $\psi_{P}$, this

$$
\begin{aligned}
& \text { \# Initial Setup - to be done in advance } \\
& \text { Determine ranges for each average and likelihood } \\
& \quad \text { and calculate look-up tables } \\
& \text { \# Note: } \beta=\sqrt{2 k T / \eta V} \\
& \#\left\langle P_{-}\right\rangle=-(\beta / \sqrt{\pi}) \exp \left(\left(-E_{t m p-} / \eta \beta\right) .^{2}\right) / \operatorname{erfc}\left(E_{t m p-} / \eta \beta\right) \\
& \#\left\langle P_{+}\right\rangle=(\beta / \sqrt{\pi}) \exp \left(\left(-E_{t m p+} / \eta \beta\right) .^{2}\right) / \operatorname{erfc}\left(E_{t m p+} / \eta \beta\right) \\
& \# p_{-+}=(\Delta t / \tau)\left(1-\operatorname{erfc}\left(\left(E_{t m p-} / \text { eta }+\epsilon\right) / \text { beta }\right)\right. \\
& \# \quad / \text { erfc }\left(E_{t m p-} / \text { eta } \beta\right) \text { for } E_{e}<E_{c} \\
& \# p_{+-}=(\Delta t / \tau)\left(1-\operatorname{erfc}\left(\left(-E_{t m p+} / \text { eta }+\epsilon\right) / \text { beta }\right)\right. \\
& \# \quad / \text { erfc }\left(-E_{t m p+} / \text { eta } \beta\right) \text { for } E_{e}>-E_{c}
\end{aligned}
$$$$
\text { \# Note: we assume a single stepsize and } E_{e} \text { range for }
$$$$
\text { \# brevity. This is not the best choice for accuracy, but the }
$$$$
\text { \# extension is straightforward. }
$$$$
\text { For }\left(i=0 \ldots N_{c}-1\right), E_{c}[i]=E_{c}[i] / E_{\text {step }} \text { End For }
$$$$
\text { For }\left(j=0 \ldots N_{I}-1\right), E_{I}[j]=E_{I}[j] / E_{\text {step }} \text { End For }
$$$$
w_{\text {sum }}=\sum_{i=0}^{N_{c}-1} \quad \sum_{j=0}^{N_{I}-1} w_{c}[i] w_{I}[j] \quad / \eta
$$$$
\text { addit }=\sum_{i=0}^{N_{c}-1} \quad \sum_{j=0}^{N_{I}-1} w_{c}[i] w_{I}[j] E_{I}[j] \quad / \eta-P_{R} \eta w_{\text {sum }}
$$$$
P_{R}=2 P_{R}
$$

$$
\begin{aligned}
& \text { \# Begin Iteration } \\
& \text { For } k=0 \ldots \text { length }(E)-1 \\
& \begin{aligned}
P[k]=E[k] w_{\text {sum }}+\text { addit } \\
\text { base }=\left(E[k]-E_{\text {effmin }}\right) / E_{\text {step }}
\end{aligned} \\
& \text { For } j=0 \ldots N_{I}-1 \\
& \qquad \begin{aligned}
& \text { out }=0 \\
& \text { base } 2=\text { base }+E_{I}[j] \\
& \text { For } i=0 \ldots N_{c}-1 \\
& h_{-}=\operatorname{round}\left(\text { base } 2-E_{c}[i]\right) \\
& h_{+}=\operatorname{round}\left(\text { base } 2+E_{c}[i]\right) \\
& x_{+} {[i, j]=\left(x_{+}[i, j]+p_{-+}\left[h_{-}\right]\right) /\left(1+p_{-+}\left[h_{-}\right]+p_{+-}\left[h_{+}\right]\right) } \\
& \text {out }=\text { out }+w_{c}[i]\left(x_{+}[i, j]\left(P_{\text {pos }}\left[h_{+}\right]-P_{n e g}\left[h_{-}\right]+P_{R}\right)\right. \\
&\left.+P_{\text {neg }}\left[h_{-}\right]\right)
\end{aligned}
\end{aligned}
$$

End For

$$
P[k]=P[k]+w_{I}[j] \text { out }
$$

End For

End For

Algorithm 2: Implementation algorithm for the model with thermal relaxation but negligible stress-dependence. For brevity, all step sizes and value ranges are treated as equal. 
effectively yields three different polynomials to minimize corresponding to the three possible dipole orientations. We perform the minimization corresponding to each state in advance and store it in a lookup table. In this case there are two independent variables in (11) that cannot be combined: the input field $E_{e}$ and input stress $\sigma$. Thus, this formulation requires three $2-\mathrm{D}$ tables.

As in previous algorithms, memory can be conserved by noting that some values need not be computed. For values of $E_{e}<-E_{c}\left[N_{c}\right]$, the minima corresponding to positive orientations need not be computed because the dipole will have always switched to a different orientation. Likewise negative orientations need not be computed for $E_{e}>E_{c}\left[N_{c}\right]$, and the ninety degree orientation does not need computed for values at either extreme. This reduces the memory requirement of the table. Implementation of this approach is detailed in Algorithm 3.

Computation in the real-time portion of the algorithm is independent of lookup table size, as in Algorithm 2. However, this algorithm also possesses a way to trade a small amount of computation time for increased accuracy, while holding the table size constant. The second derivative of the energy is small - i.e., the minimized energy corresponding to each dipole state is nearly linear. Much better accuracy can be obtained by linearly interpolating between the table values. Since these values occupy a $2-\mathrm{D}$ grid, this is efficiently approximated by interpolating

\# Initial Setup - to be done in advance

Calculate the minimum Gibb's energy tables at the given

$E_{e}$ and $\sigma$ values for each possible orientation.

Call these $P_{\text {pos }}, P_{90}$, and $P_{n e g}$

c90posconst $=P_{I}\left(2 q^{2} P_{I}^{2}-3 a q P_{I}+a^{2}-\eta Y\right) / Y+\eta P_{R}$

c90poscoeff $=\left(2 q P_{I}-a\right) / Y$

c90posconst $=-P_{I}\left(2 q^{2} P_{I}^{2}+3 a q P_{I}+a^{2}-\eta Y\right) / Y-\eta P_{R}$

c90poscoeff $=-\left(2 q P_{I}+a\right) / Y$

For $\left(j=0 \ldots N_{I}-1\right), E_{I}[j]=E_{I}[j] / E_{\text {step }}$ End For

\# Begin Iteration

For $k=0 \ldots \operatorname{length}(E)-1 \# \operatorname{length}(E)=\operatorname{length}(\sigma)$

$c 90$ pos $=c 90$ poscoef $f \sigma[k]+c 90$ posconst

$c 90$ neg $=c 90$ negcoeff $\sigma[k]+c 90$ negconst

$P[k]=0$

$\sigma_{\text {ind }}=\operatorname{round}\left(\left(\sigma[k]-\sigma_{\text {min }}\right) / \sigma_{\text {step }}\right)$

$E_{\text {postmp }}=\left(E[k]-E_{\text {posmin }}\right) / E_{\text {step }}$

$E_{90 t m p}=\left(E[k]-E_{\text {ninetymin }}\right) / E_{\text {step }}$

$E_{\text {negtmp }}=\left(E[k]-E_{\text {negmin }}\right) / E_{\text {step }}$

For $j=0 \ldots N_{I}-1$

out $=0$

$E_{e}=E[k]+E_{I}[j]$

$E_{\text {posind }}=\operatorname{round}\left(E_{\text {postmp }}+E_{I}[j]\right)$

$E_{90 i n d}=\operatorname{round}\left(E_{\text {ninetytmp }}+E_{I}[j]\right)$

$E_{\text {negind }}=\operatorname{round}\left(E_{\text {negtmptmp }}+E_{I}[j]\right)$

For $i=0 \ldots N_{c}-1$

If $\delta[i, j]==1 \#$ positive

If $E_{e}<=c 90 n e g$ and $E_{e}>-E_{c}[i]$

$\delta[i, j]=0$

out $=$ out $+w_{c}[i] P_{90}\left[E_{90 i n d}, \sigma_{\text {ind }}\right]$

Else If $E_{e} \leq-E_{c}[i]$

$$
\begin{aligned}
& \delta[i, j]=-1 \\
& \text { out }=\text { out }+w_{c}[i] P_{n e g}\left[E_{\text {negind }}, \sigma_{\text {ind }}\right] \\
& \text { lse } \\
& \text { out }=\text { out }+w_{c}[i] P_{\text {pos }}\left[E_{\text {posind }}, \sigma_{\text {ind }}\right]
\end{aligned}
$$$$
\text { Else }
$$

End If

Else If $\delta[i, j]==0 \# 90$ degree

If $E_{e} \geq E_{c}[i]$

$$
\delta[i, j]=1
$$$$
\text { out }=\text { out }+w_{c}[i] P_{\text {pos }}\left[E_{\text {posind }}, \sigma_{\text {ind }}\right]
$$

Else If $E_{e} \leq-E_{c}[i]$

$$
\begin{aligned}
& \delta[i, j]=-1 \\
& \text { out }=\text { out }+w_{c}[i] P_{\text {neg }}\left[E_{\text {negind }}, \sigma_{\text {ind }}\right]
\end{aligned}
$$

Else

$$
\text { out }=\text { out }+w_{c}[i] P_{90}\left[E_{90 i n d}, \sigma_{\text {ind }}\right]
$$

End If

Else \# 90 negative

$$
\begin{aligned}
& \text { If } E_{e}>=c 90 \text { pos and } E_{e}<E_{c}[i] \\
& \quad \delta[i, j]=0 \\
& \quad \text { out }=\text { out }+w_{c}[i] P_{90}\left[E_{90 i n d}, \sigma_{\text {ind }}\right] \\
& \text { Else If } E_{e} \geq E_{c}[i] \\
& \quad \delta[i, j]=1 \\
& \quad \text { out }=\text { out }+w_{c}[i] P_{\text {pos }}\left[E_{\text {posind }}, \sigma_{\text {ind }}\right] \\
& \text { Else } \\
& \quad \text { out }=\text { out }+w_{c}[i] P_{\text {neg }}\left[E_{\text {negind }}, \sigma_{\text {ind }}\right]
\end{aligned}
$$

End If

End If

End For

$P[k]=P[k]+w_{I}[j]$ out

End For

End For

Algorithm 3: Implementation algorithm for model with stress-dependence but negligible relaxation. 
along one dimension and then the other. Allowing $E_{1}$ and $\sigma_{1}$ to represent the closest table value below $E_{e}$ and $\sigma$, and $E_{2}, \sigma_{2}$ to be the values just above the desired value, this can be written in one equation as

$$
\begin{aligned}
\langle P\rangle= & P_{11}\left(1-\frac{E_{e}-E_{1}}{E_{\text {step }}}\right)\left(1-\frac{\sigma-\sigma_{1}}{\sigma_{\text {step }}}\right)+P_{12} \frac{E_{e}-E_{1}}{E_{\text {step }}}\left(1-\frac{\sigma-\sigma_{1}}{\sigma_{\text {step }}}\right) \\
& +P_{21}\left(1-\frac{E_{e}-E_{1}}{E_{\text {step }}}\right) \frac{\sigma-\sigma_{1}}{\sigma_{\text {step }}}+P_{22} \frac{\left(E_{e}-E_{1}\right)\left(\sigma-\sigma_{1}\right)}{E_{\text {step }} \sigma_{\text {step }}} .
\end{aligned}
$$

Here $\langle P\rangle=\left\langle P_{-}\right\rangle,\left\langle P_{90}\right\rangle$ or $\left\langle P_{+}\right\rangle$, values from the table are given by $P_{11}=\langle P\rangle\left(E_{1}, \sigma_{1}\right), P_{12}=\langle P\rangle\left(E_{1}, \sigma_{2}\right)$, etc., $E_{\text {step }}$ is the distance between two field values in the table, and $\sigma_{\text {step }}$ the distance between stress values. In the numerical tests detailed in Section 4, this was found to increase the accuracy by 2 to 3 orders of magnitude without significantly altering the computation time.

\subsection{Algorithm for Thermal Relaxation and Stress-Dependent Regimes}

The use of a look-up table becomes less effective when relaxation and stress-dependent effects are both included. Here, the average polarizations and switching likelihoods depend on $E_{e}, E_{c}$, and $\sigma$. Unlike the negligible stress case, however, the inclusion of addition terms in (11) no longer allows $E_{e}$ and $E_{c}$ to be combined into a single variable. This necessitates a $3-\mathrm{D}$ lookup table for each of the average polarizations and stress values, yielding a total of 6 tables. The $E_{c}$ dimension can be accommodated exactly by using one value at each of the quadrature points for the $\nu_{c}$ distribution, so that error is only introduced on $E_{e}$ and $\sigma$. However, this will likely require 25100 times more memory than the stress-only variant. Further, while it does not effect the real-time portion of the algorithm, calculation of the look-up table can be computationally expensive, which effects parameter estimation techniques. Nevertheless, the look-up table does provide three to four orders of magnitude improvement over a direct implementation in the real-time portion of the computations. Implementation for this case is summarized in Algorithm 4.

\# Initial Setup - to be done in advance

Determine ranges for each average and likelihood

and calculate look-up tables via quadrature

\# Note: we assume a single stepsize and $E_{e}$ range for

\# brevity. This is not the best choice for accuracy, but the

\# extension is straightforward.

For $\left(j=0 \ldots N_{I}-1\right), E_{I}[j]=E_{I}[j] / E_{\text {step }}$ End For

\# Begin Iteration

$$
\begin{aligned}
& \text { For } k=0 \ldots \text { length }(E)-1 \\
& P[k]=0 \\
& \text { avg }_{\text {tmp }}=\left(E[k]-E_{\text {effmin }}\right) / E_{\text {step }} \\
& \sigma_{\text {ind }}=\operatorname{round}\left(\sigma[k]-\sigma_{\text {min }}\right) / \sigma_{\text {step }} \\
& \text { base }=\left(E[k]-E_{\text {effmin }}\right) / E_{\text {step }} \\
& \text { For } j=0 \ldots N_{I}-1 \\
& E_{\text {ind }}=\operatorname{round}\left(\text { base }+E_{I}[j]\right)
\end{aligned}
$$

$$
\begin{aligned}
& \text { out }=0 \\
& \text { For } i=0 \ldots N_{c}-1 \\
& \alpha_{+}=1+p_{90+}\left[E_{\text {ind }}, \sigma_{\text {ind }}, i\right]+p_{+90}\left[E_{\text {ind }}, \sigma_{\text {ind }}, i\right] \\
& \alpha_{-}=1+p_{90-}\left[E_{i n d}, \sigma_{i n d}, i\right]+p_{-90}\left[E_{\text {ind }}, \sigma_{i n d}, i\right] \\
& x_{+}[i, j]=\left(\alpha_{-} x_{+}[i, j]+p_{90+}\left[E_{\text {ind }}, \sigma_{\text {ind }}, i\right]\left(\alpha_{-}\right.\right. \\
& \left.\left.-x_{-}[i, j]-p_{90-}\left[E_{\text {ind }}, \sigma_{\text {ind }}, i\right]\right)\right) /\left(\alpha_{+} \alpha_{-}\right. \\
& \left.-p_{90+}\left[E_{\text {ind }}, \sigma_{\text {ind }}, i\right] p_{90-}\left[E_{\text {ind }}, \sigma_{\text {ind }}, i\right]\right) \\
& x_{-}[i, j]=\left(x_{-}[i, j]+p_{90 n}\left[E_{\text {ind }}, \sigma_{\text {ind }}, i\right]\left(1-x_{+}[i, j]\right)\right) / \alpha_{-} \\
& \text {out }=\text { out }+w_{c}[i]\left(x _ { - } [ i , j ] \left(P_{\text {neg }}\left[E_{\text {ind }}, \sigma_{\text {ind }}, i\right]\right.\right. \\
& \left.-P_{90}\left[E_{i n d}, \sigma_{i n d}, i\right]\right)+x_{+}[i, j]\left(P_{\text {pos }}\left[E_{\text {ind }}, \sigma_{\text {ind }}, i\right]\right. \\
& \left.\left.-P_{90}\left[E_{\text {ind }}, \sigma_{\text {ind }}, i\right]\right)+P_{9} 0\left[E_{\text {ind }}, \sigma_{\text {ind }}, i\right]\right)
\end{aligned}
$$

End For

$P[k]=P[k]+w_{I}[j]$ out

End For

End For

Algorithm 4: Implementation algorithm for the model with relaxation and stress-dependence. All step sizes and value ranges in the look-up table are treated as equal for brevity. 


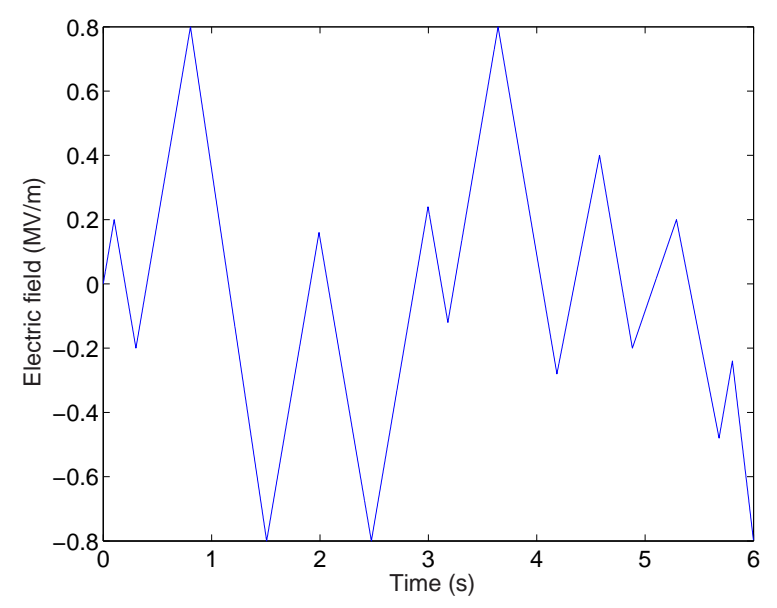

(a)

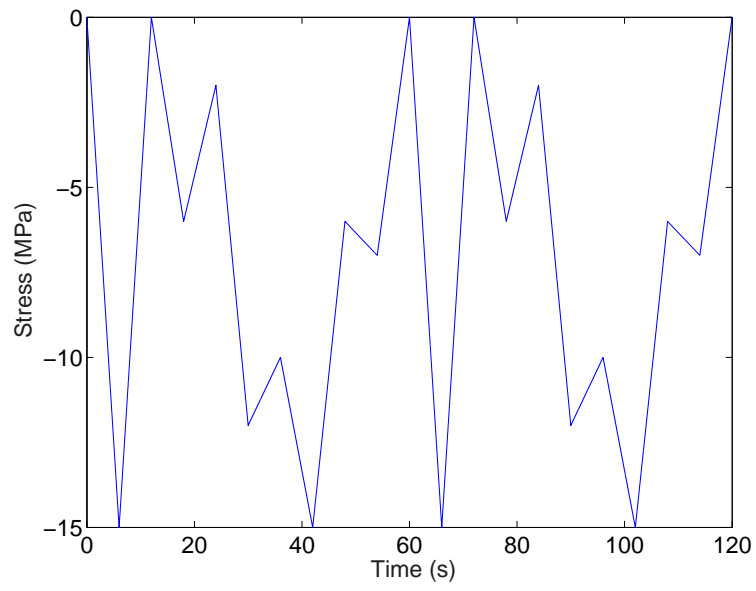

(b)

Figure 4: Inputs used for the direct implementation of the model and Algorithms 1-4 based on lookup tables. (a) Input electric field - this pattern repeated 20 times, and (b) Input stress, where applicable

\section{Lookup Table Approximation Run-times and Error}

Compared to the direct "exact" implementation of the homogenized energy model, the lookup table (LUT) implementations yield significantly better performance. Algorithms 1-4 were implemented using the same parameters and iterations as the direct implementations given in Table 1. All computations were performed using a Pentium IV Xeon 1.7 GHz processor running Linux. Algorithms 1-4 were implemented in a C language MATLAB mex file (available from http://research.coire.net). The run-times for the input shown in Figure 4 are given in Table 2. For each of these tests, the lookup table size was limited to 1000 elements per table for Algorithms 2 and 3 and 80,000 elements per table -1000 for $E_{e} \times \sigma$ times 80 values for $E_{c}-$ for Algorithm 4 . In all cases, the implementation algorithms employed the parameters given in [2]. When reporting results, we let $N_{\langle P\rangle}$ denote the number of quadrature points used when approximating the average polarizations $\left\langle P_{-}\right\rangle,\left\langle P_{90}\right\rangle,\left\langle P_{+}\right\rangle$in $(17)$ or (21) and $N_{p}$ denote the number of quadrature points employed for the likelihoods $p_{-+}, p_{+-}, p_{-90}, p_{90-}, p_{+90}$, $p_{90+}$ in $(19)$ or $(23)$

We first compare the run-times for the lookup table approximations with the direct implementation. Algorithm 1 ran in one-third of the time of the unoptimized version on this platform whereas Algorithm 2 ran almost 50 times faster than a direct implementation. With stress-dependence, Algorithm 3 ran 10-12 times faster (depending on whether the setup time is included) and Algorithm 4 ran either 4000 or 13,800 times faster, again depending on whether or not setup time is included in the comparison. Thus, the lookup table approximations

\begin{tabular}{l|rr}
\hline Algorithm & Setup time $(\mathrm{s})$ & Run-time $(\mathrm{s})$ \\
\hline Neg. relaxation, no stress & 0 & 9.03 \\
Relaxation, no stress & 0.09 & 50.36 \\
Neg. relaxation, stress dep. ( $\sigma$ constant) & 0.09 & 10.50 \\
Neg. relaxation, stress dep. $(\sigma$ varying) & 0.08 & 10.91 \\
Relaxation, stress dep. $(\sigma$ constant) & 12.64 & 56.40 \\
Relaxation, stress dep. $(\sigma$ varying) & 122.64 & 57.12 \\
\hline
\end{tabular}

Table 2: Setup time and run-times for Algorithms 1-4, computed on a Pentium IV 1.7 GHz processor running Linux, with the algorithms implemented as C language MATLAB mex files and 120,000 time steps being computed. 


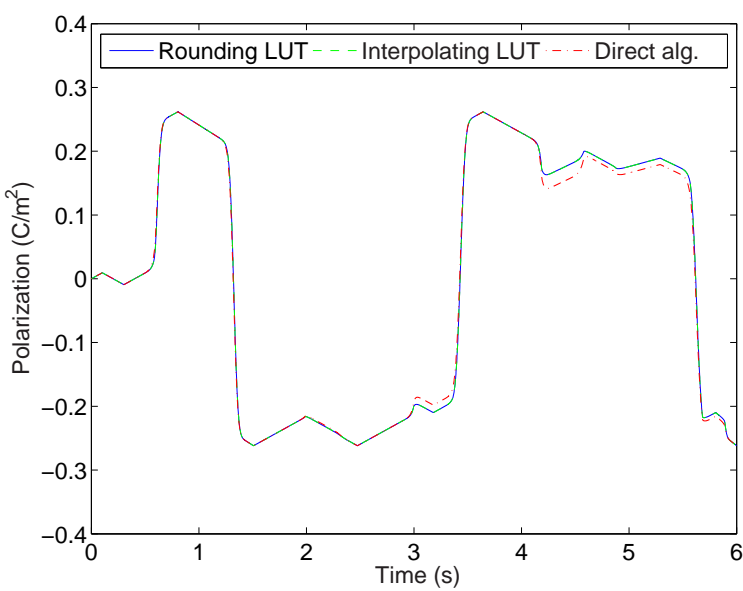

(a)

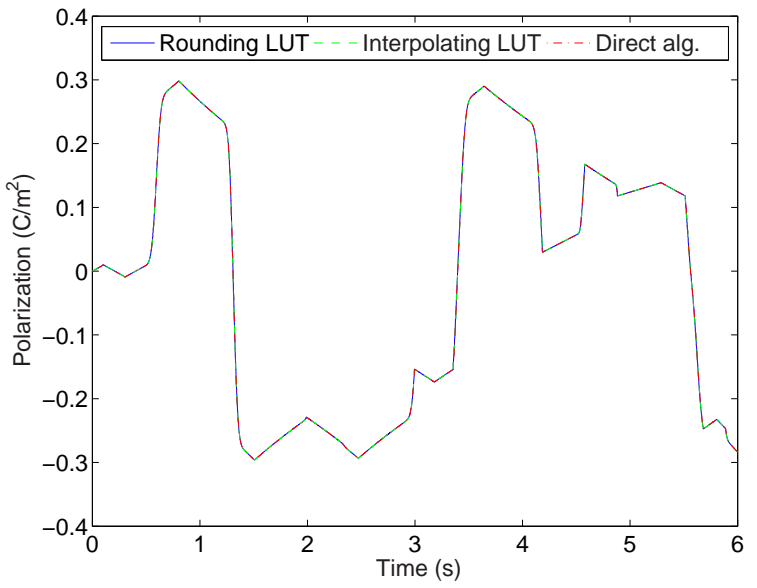

(c)

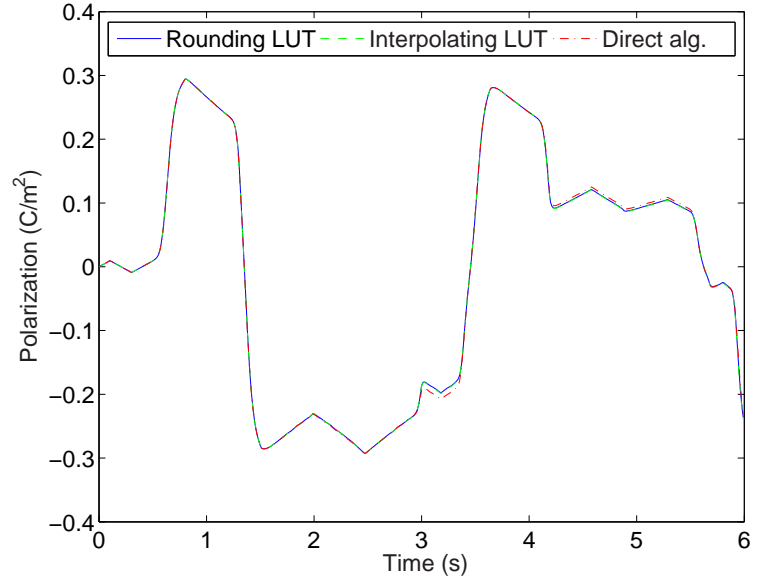

(b)

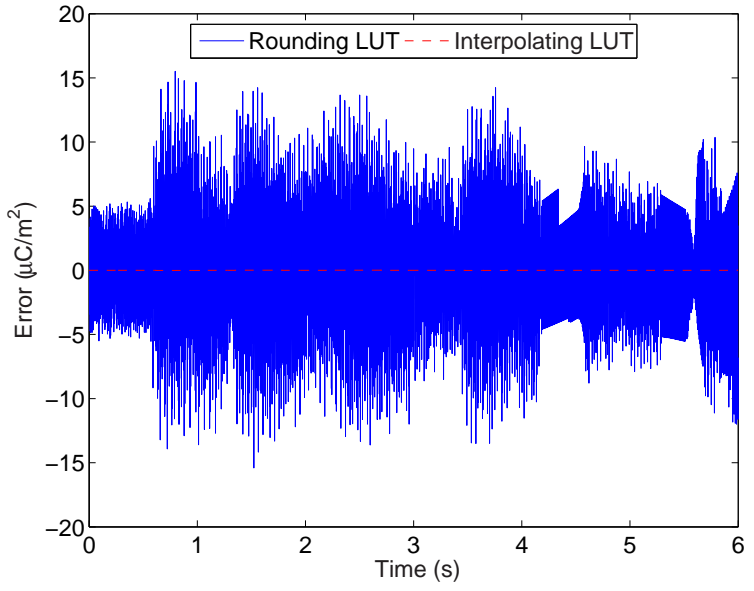

(d)

Figure 5: Comparisons of polarization given by direct model implementation and algorithms based on lookup tables (LUT). (a) Stress-invariant, relaxation algorithm (Algorithm 2). (b) Stress-dependent, relaxation algorithm (Algorithm 4). (c) Stress-dependent negligible relaxation algorithm (Algorithm 3) and (d) approximation error with and without interpolation.

yield anywhere from 1 to 4 orders of magnitude improvement depending on which formulation is employed.

The level of accuracy afforded by the lookup tables can be adjusted without altering the run-time, although memory usage and setup time increases with accuracy. To illustrate this, Algorithm 2 was compared with a direct implementation of the stress-invariant relaxation model using parameters taken from [2]. The results are summarized in Table 3 and Figure $5(\mathrm{a})$. The number of quadrature points $N_{\langle P\rangle}$ and $N_{p}$ were adjusted separately. The table suggests that memory is best used by allowing larger tables for likelihoods than average polarizations. We note that computation time does not increase as the table size increases. Surprisingly, the table does not show setup time increases as memory increases. This is somewhat misleading since the calculation is rapid, and the clock on the computer does not have sufficient resolution to resolve the differences as memory increases.

The trends for Algorithm 3 are similar and yield the errors summarized in Table 4 . In this case, we include error values for both rounding and interpolating. Setup time does increase as the amount of memory increases. Since the lookup tables are two dimensional, the number of points used for both the input field and input stress are given. In addition to noting the consistent run-times for all table sizes, we note that interpolation did not increase the run-time of the algorithm. This is surprising in the sense that interpolation requires two to four times 


\begin{tabular}{rrrrr}
\hline$N_{\langle P\rangle}$ & $N_{p}$ & Setup time $(\mathrm{s})$ & Run-time (s) & RMS error $(\%)$ \\
\hline 500 & 500 & 0.06 & 54.12 & 3.229 \\
500 & 1000 & 0.07 & 53.67 & 3.047 \\
500 & 2000 & 0.07 & 55.37 & 2.967 \\
1000 & 500 & 0.06 & 53.43 & 3.230 \\
1000 & 1000 & 0.06 & 54.13 & 3.048 \\
1000 & 2000 & 0.07 & 55.78 & 2.968 \\
2000 & 500 & 0.07 & 53.80 & 3.230 \\
2000 & 1000 & 0.07 & 54.54 & 3.049 \\
2000 & 2000 & 0.07 & 55.87 & 2.969 \\
\hline
\end{tabular}

Table 3: Error between the direct model implementation and Algorithm 2 for regimes with thermal relaxation but negligable stress-dependencies. Errors are root mean square (RMS) values expressed as percentages of the saturation polarizations $\left(0.3 \mathrm{C} / \mathrm{m}^{2}\right)$. Run-times are for 120,000 time steps.

the memory access and adds several additional additions and multiplies to each kernel evaluation. Investigation showed the computation had a bottleneck with the storage of $\delta$ values to memory and apparently the extra computation required for interpolation was performed when the processor would otherwise have been waiting for access. This is both an architecture and a compiler-dependent feature, and the same performance may not be seen on other platforms. However, the accuracy gained by interpolation, 2 to 3 orders of magnitude, is not architecture dependent. It will vary if different material parameters are employed but the qualitative behavior should remain regardless of architecture. As such, interpolation provides an effective way to limit lookup table size while improving computation performance. These results are also plotted in Figure $5(\mathrm{c})$ and (d) to provide a comparison to the direct implementation and illustrate the error introduced by the lookup table approximation.

Given the accuracy gained by interpolation, it is reasonable to ask why it is not performed on Algorithm 2 or 4 . The likelihoods (19) and (23) are exponential; linear interpolation as employed for Algorithm 3 will overpredict the likelihoods, yielding moments that switch too soon. Each moment that switches prematurely adds $2 P_{R}$ to the approximation error. As such, linear interpolation does not yield a significant increase when relaxation effects are included. This also explains the slower convergence of the approximations including thermal relaxation; small errors present in the likelihood calculation dictate small errors in the dipole states which give larger errors in the output polarization.

Table 5 and Figure 5(b) illustrate the accuracy of Algorithm 4 for regimes which include both stressdependence and relaxation. In terms of accuracy and run-time, the results are comparable to Algorithms 2 and 3. However, in terms of setup time, this algorithm is significantly slower and uses significantly more mem-

\begin{tabular}{rrr|rr|rr}
\hline & & \multicolumn{2}{|c|}{ Rounding } & \multicolumn{2}{|c}{ Interpolating } \\
Field points & Stress points & Setup time (s) & Run-time (s) & error (\%) & Run-time (s) & error (\%) \\
\hline 125 & 125 & 0.95 & 10.36 & 0.01258 & 10.61 & $9.26 \times 10^{-5}$ \\
250 & 125 & 1.52 & 10.45 & 0.00922 & 10.44 & $2.53 \times 10^{-5}$ \\
500 & 125 & 3.04 & 10.34 & 0.00907 & 10.45 & $8.71 \times 10^{-6}$ \\
125 & 250 & 1.84 & 10.42 & 0.01000 & 10.45 & $9.03 \times 10^{-5}$ \\
250 & 250 & 10.38 & 0.00514 & 10.40 & $2.30 \times 10^{-5}$ \\
500 & 250 & 1.67 & 10.49 & 0.00487 & 10.70 & $6.26 \times 10^{-6}$ \\
125 & 500 & 4.89 & 10.60 & 0.00922 & 10.66 & $8.97 \times 10^{-5}$ \\
250 & 500 & 9.79 & 10.50 & 0.00342 & 10.71 & $2.24 \times 10^{-5}$ \\
500 & 500 & 19.71 & 10.56 & 0.00298 & 10.63 & $5.67 \times 10^{-6}$ \\
\hline
\end{tabular}

Table 4: Comparison of the direct and look-up table stress-dependent, negligible relaxation Algorithm 3. Errors are root mean square values expressed as percentages of the saturation polarizations $\left(0.3 \mathrm{C} / \mathrm{m}^{2}\right)$. Run-times are for 120,000 time steps. 


\begin{tabular}{rrrrr}
\hline Field points & Stress points & Setup time (s) & Run-time (s) & RMS Error \\
\hline 25 & 25 & 107.20 & 0.28 & 2.42363 \\
50 & 25 & 173.97 & 0.28 & 1.47033 \\
100 & 25 & 307.18 & 0.29 & 1.16744 \\
25 & 50 & 215.55 & 0.28 & 2.52963 \\
50 & 50 & 343.12 & 0.28 & 1.21354 \\
100 & 50 & 615.48 & 0.29 & 1.06211 \\
25 & 100 & 426.51 & 0.28 & 2.53719 \\
50 & 100 & 693.13 & 0.28 & 1.21641 \\
100 & 100 & 1225.09 & 0.29 & 1.06611 \\
\hline
\end{tabular}

Table 5: Comparison of the direct and look-up table for the stress-dependent, relaxation Algorithm 4, with a varying input stress and $80 E_{c}$ quadrature points. Errors are root mean square values expressed as percentages of the saturation polarizations $\left(0.3 \mathrm{C} / \mathrm{m}^{2}\right)$. Run-times are for 600 time steps.

ory. This is an unavoidable attribute of the method. Note that Table 5 shows run-times for only 600 temporal iterations. This amount was chosen to keep the run-time of the direct implementation algorithm manageable. As shown in Table 2, Algorithm 4 could complete 120,000 iterations in just under a minute, not including the setup time for the desired table size.

\section{Comparison to Experimental Data}

In Section 4, we demonstrate that through the use of lookup tables, the model formulations which incorporate thermal relaxation and stress effects can be implemented in a highly efficient manner while maintaining the accuracy of the original model. We summarize here the performance of the framework, as implemented using the algorithms developed in Section 3, for characterizing the magnetic after-effects and stress-dependencies illustrated in Figure 1. We note that the unified nature of the framework permits a direct extension of Algorithms 1-4 to ferromagnetic regimes.

In Figure 6, we illustrate the capability of the magnetic model, implemented via Algorithm 2, to characterize data collected from a cylindrical steel rod having a length of 2 inches and diameter of 0.25 inches. Specifically, it is noted that by incorporating relaxation mechanisms, the model characterizes the magnetic after-effects which yield negative differential susceptibilities following field reversal at the beginning of minor loops. Additional details regarding both the experimental setup and model performance are provided in [3].

The performance of the stress-dependent polarization model, implemented via Algorithm 3, is illustrated in Figure 7 through comparison with PLZT data from [9]. Parameters were estimated through a least-squares fit to data collected at prestress levels of $\sigma_{0}=0 \mathrm{MPa}, \sigma_{0}=-6 \mathrm{MPa}, \sigma_{0}=-10 \mathrm{MPa}$ and $\sigma_{0}=-15 \mathrm{MPa}$ to obtain the present model fits. The fits in (b) and (d) illustrate minor loop attributes of the data and model near negative remanence. The change in curvature exhibited by the data with $\sigma_{0}=-15 \mathrm{MPa}$ illustrates that the manifestation of $90^{\circ}$ switching becomes increasingly prominent as prestress levels increase. This illustrates the necessity of incorporating $90^{\circ}$ switching mechanisms in both the model and implementation algorithms. Additional details regarding the experimental setup can be found in [9] whereas the model extensions required to address $90^{\circ}$ switching are presented in $[1,2]$.

\section{Concluding Remarks}

The homogenized energy model developed in $[11,16,18,20]$ provides a unified framework for characterizing hysteresis and constitutive nonlinearities in ferroelectric, ferromagnetic and ferroelastic materials. Due to the energy formulation of the framework, the effects of thermally activated relaxation phenomenon, stress-dependence and thermal dependence can be incorporated in a natural manner. However, direct implementation algorithms for models that incorporate these mechanisms presently do not provide the efficiency required for high-speed material 


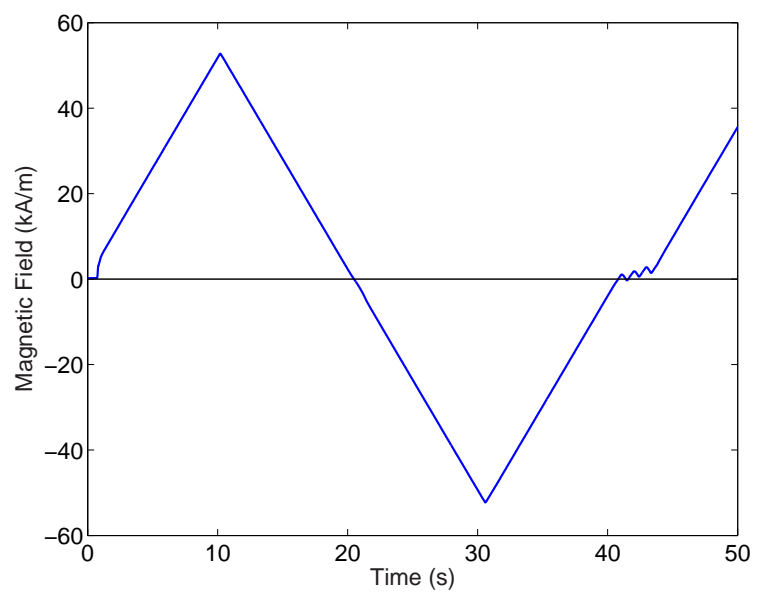

(a)

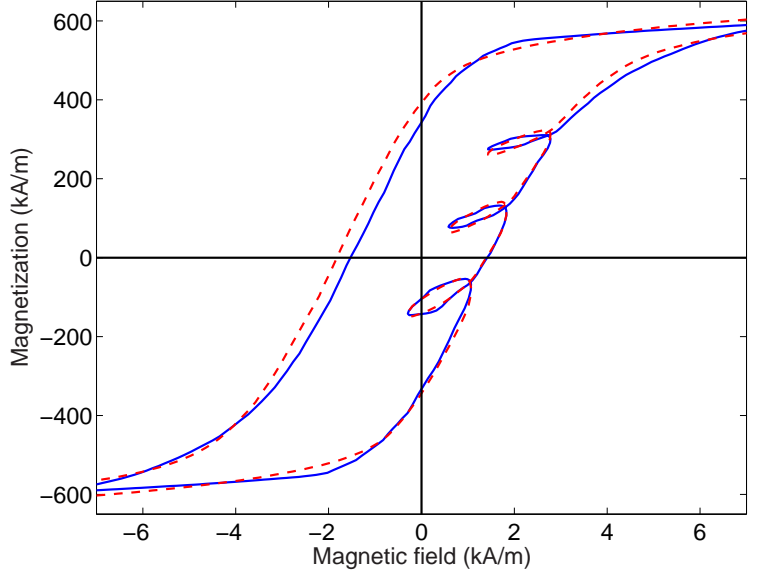

(b)

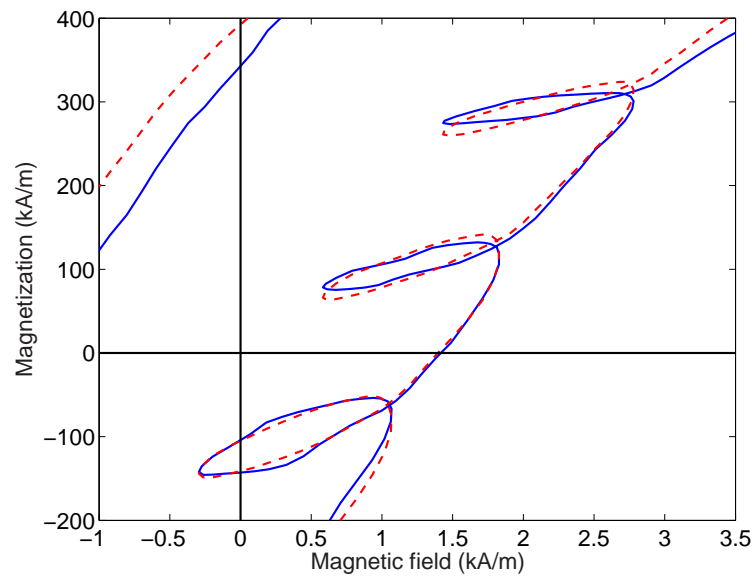

(c)

Figure 6: (a) Input magnetic field, (b) fit (-) provided by Algorithm 2 to magnetization data (- - ) from [3], and (c) detailed view of fit.

characterization, actuator or sensor optimization, or real-time control implementation. In this paper, we present highly efficient implementation algorithms, based on lookup tables, which reduce implementation times by one to four orders of magnitude for models which incorporate thermal activation and stress-dependence.

In addition to providing significant improvements in efficiency, the use of lookup table approximations hold a further advantage for those using embedded devices - either microprocessor or gate-array based - in which integer arithmetic is required. The calculation of minimum energies, average polarizations, and likelihoods may involve computation on very large or very small numbers. By moving these calculations offline, they may be performed within a processor capable of floating point operations and returned to integer or fixed-point format for storage and real-time processing.

As detailed in $[8,14]$, a highly advantageous attribute of the homogenized energy framework is the property that approximate model inverses can be constructed with nearly the same efficiency as the direct models. This permits the design of inverse filters which approximately linearize hysteretic actuator or sensor dynamics and hence permit linear control design. The development and experimental implementation of inverse filters based on the algorithms presented here is under investigation. 


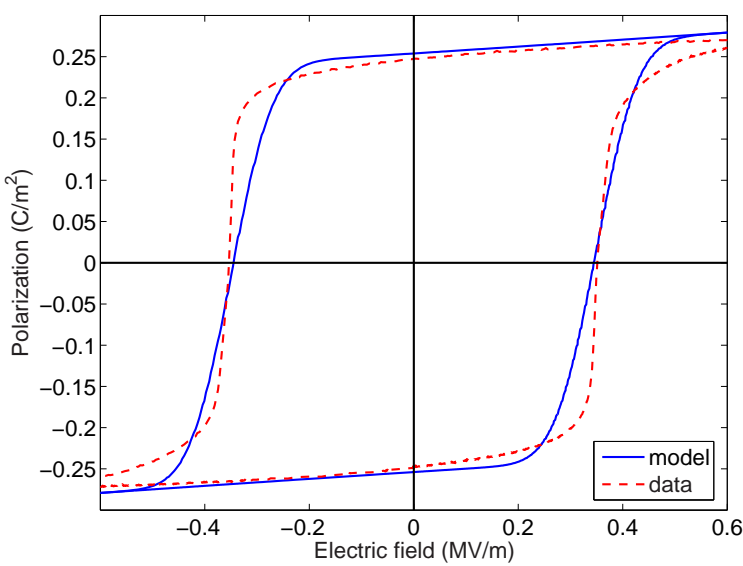

(a)

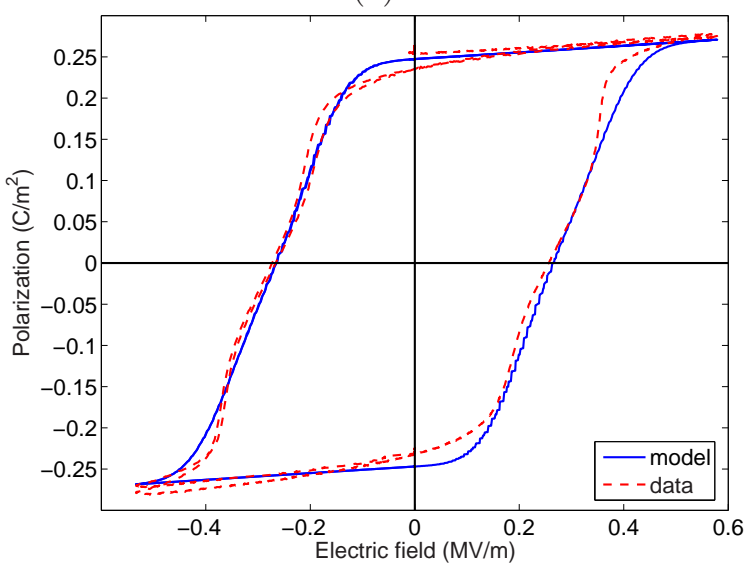

(c)

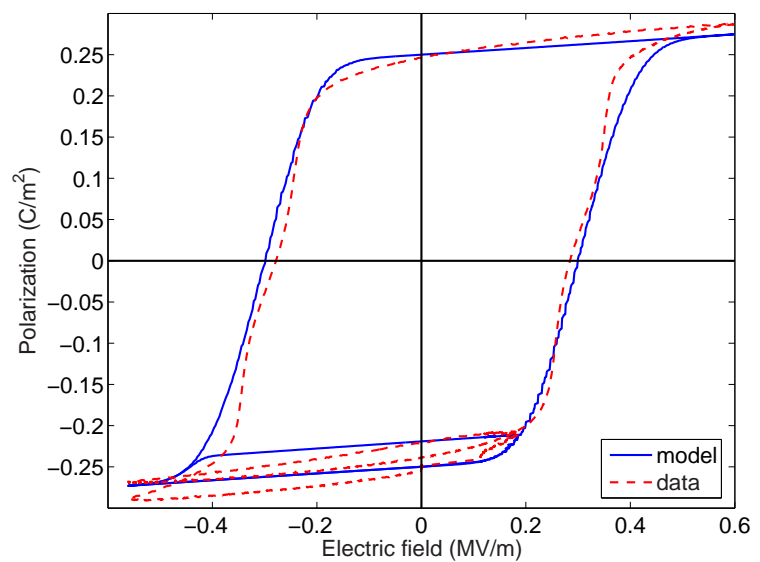

(b)

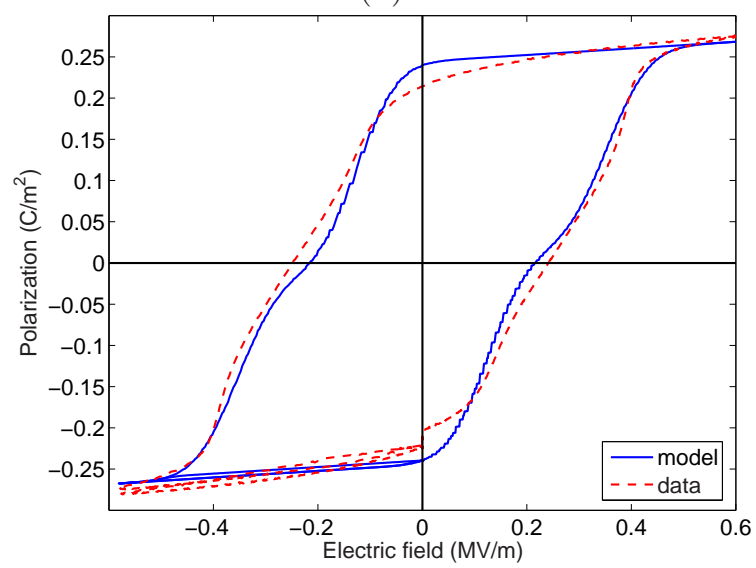

(d)

Figure 7: Comparison of modeled $E-P$ implemented in Algorithm 3 with PLZT data from [9] for compressive prestresses of (a) $\sigma_{0}=0 \mathrm{MPa}$, (b) $\sigma_{0}=-6 \mathrm{MPa}$, (c) $\sigma_{0}=-10 \mathrm{MPa}$ and (d) $\sigma_{0}=-15 \mathrm{MPa}$.

\section{ACKNOWLEDGMENTS}

The research of T.R.B. was supported by the United States Department of Education through a GAANN fellowship. The research of R.C.S. was supported in part through the NSF grant CMS-0201560 and in part by the Air Force Office of Scientific Research through the grant AFOSR-FA9550-04-1-0203.

\section{References}

[1] B.L. Ball, R.C. Smith, S. Kim, and S. Seelecke, "A ferroelectric switching model for lead zirconate titanate (PZT)," Smart Structures and Materials 2005, Proceedings of the SPIE, Volume 5757, pp. 1-9, 2005.

[2] B.L. Ball, R.C. Smith, S. Kim, and S. Seelecke, "A stress-dependent hysteresis model for ferroelectric materials," CRSC Technical Report CRSC-TR05-17. Journal of Intelligent Material Systems and Structures, submitted.

[3] T.R. Braun, R.C. Smith, and M.J. Dapino, "Experimental validation of a homogenized energy model for magnetic after-effects." CRSC Technical Report CRSC-TR05-26. Applied Physics Letters, submitted.

[4] E. Della Torre, Magnetic Hysteresis, IEEE Press, New York, 1999. 
[5] P. Ge and M. Jouaneh, "Modeling hysteresis in piezoceramic actuators," Precision Engineering, 17, pp. 211$221,1995$.

[6] D.C. Jiles, "Theory of the magnetomechanical effect," Journal of Physics D: Applied Physics, 28, pp. 15371546, 1995.

[7] D.C. Jiles and D.L. Atherton, "Theory of ferromagnetic hysteresis", Magnetism and Magnetic Materials, 61, pp. 1265-1281, 1984.

[8] A.G. Hatch, R.C. Smith, T. De and M.V. Salapaka, "Construction and experimental implementation of a model-based inverse filter to attenuate hysteresis in ferroelectric transducers," CRSC Technical Report CRSC-TR05-06; IEEE Transactions on Control Systems Technology, submitted.

[9] C.S. Lynch, "The effect of uniaxial stress on the elctro-mechanical response of 8/65/35 PLZT," Acta Materialia, 44(10), pp. 4137-4148, 1996.

[10] J.E. Massad and R.C. Smith, "A domain wall model for hysteresis in ferroelastic materials," Journal of Intelligent Material Systems and Structures, 14(7), pp. 455-471, 2003.

[11] J.E. Massad and R.C. Smith, "A homogenized free energy model for hysteresis in thin-film shape memory alloys," Thin Solid Films, 489(1-2), pp. 266-290, 2005.

[12] I.D. Mayergoyz, Mathematical Models of Hysteresis, Springer-Verlag, New York, 1991.

[13] G. Robert, D. Damjanovic and N. Setter, "Preisach modeling of piezoelectric nonlinearity in ferroelectric ceramics," Journal of Applied Physics, 89(9), pp. 5067-5074, 2001.

[14] R.C. Smith, Smart Material Systems: Model Development, SIAM, Philadelphia, 2005.

[15] R.C. Smith, M.J. Dapino, T.R. Braun, and A.P. Mortensen, "A homogenized energy framework for ferromagnetic hysteresis," CRSC Technical Report CRSC-TR05-08. IEEE Transactions on Magnetics, submitted.

[16] R.C. Smith, M.J. Dapino and S. Seelecke, "A free energy model for hysteresis in magnetostrictive transducers," Journal of Applied Physics, 93(1), pp.458-466, 2003.

[17] R.C. Smith and J.E. Massad, "A unified methodology for modeling hysteresis in ferroic materials," Proceedings of the 2001 ASME Design Engineering Technical Conferences and Computer and Information in Engineering Conference, Vol 6, Pt B, pp. 1389-1398, 2001.

[18] R.C. Smith, S. Seelecke, M.J. Dapino and Z. Ounaies, "A unified model for hysteresis in ferroic materials," Smart Structures and Materials 2003, Proceedings of the SPIE, Volume 5049, pp. 99-99, 2003.

[19] R.C. Smith, S. Seelecke, M.J. Dapino and Z. Ounaies, "A unified framework for modeling hysteresis in ferroic materials," CRSC Technical Report CRSC-TR04-35; Journal of Mechanics and Physics of Solids, to appear.

[20] R.C. Smith, S. Seelecke, Z. Ounaies and J. Smith, "A free energy model for hysteresis in ferroelectric materials," Journal of Intelligent Material Systems and Structures, 14(11), pp. 719-739, 2003.

[21] G. Webb, A. Kurdila and D.C. Lagoudas, "Adaptive hysteresis model for model reference control with actuator hysteresis," Journal of Guidance, Control and Dynamics, 23(3), pp. 459-465, 2000. 